\title{
Quercetin glucosides inhibit glucose uptake into brush-border- membrane vesicles of porcine jejunum
}

\author{
Rainer Cermak, Sandra Landgraf and Siegfried Wolffram* \\ Institute of Animal Nutrition, Physiology and Metabolism, Christian-Albrechts-University Kiel, Olshausenstrasse 40, \\ D-24098 Kiel, Germany
}

(Received 23 September 2003 - Revised 15 January 2004 - Accepted 13 February 2004)

\begin{abstract}
Recent experimental data point to an interaction of dietary flavonol monoglucosides with the intestinal Na-dependent glucose transporter 1 (SGLT1). To investigate this interaction in more detail, we performed experiments with SGLT1-containing brush-border-membrane vesicles (BBMV) from pig jejunum. The flavonol quercetin-3-O-glucoside $(\mathrm{Q} 3 \mathrm{G})$ concentration-dependently inhibited Na-dependent uptake of radioactively labelled D-glucose into BBMV. Uptake of L-leucine was not inhibited by Q3G, indicating a specific interaction of the glucoside with SGLT1. Whereas the maximal transport rate of concentration-dependent initial glucose uptake was not altered in the presence of Q3G, the constant for half-maximal glucose uptake was increased, suggesting a competitive type of inhibition of glucose uptake by Q3G. Trans-stimulation experiments suggested the transport of Q3G via SGLT1. In addition, Q3G decreased the Na-independent diffusive uptake of glucose into BBMV. Other flavonoids were also tested for their inhibitory effect on D-glucose uptake. Among the tested quercetin glycosides, only the $4^{\prime}-O$-glucoside $(\mathrm{Q} 4 \mathrm{G})$ also inhibited $\mathrm{Na}$-dependent glucose uptake into BBMV, whereas the 3-O-galactoside, the 3-O-glucorhamnoside and the aglycone quercetin itself were ineffective. Glucosides of some other flavonoid classes such as naringenin-7-O-glucoside, genistein-7- $O$-glucoside and cyanidin-3,5- $O$-diglucoside were ineffective as well. Thus, dietary quercetin monoglucosides, for example, Q3G and Q4G, have an impact on intestinal nutrient transporters such as SGLT1 and related systems.
\end{abstract}

Flavonoids: Quercetin monoglucosides: Intestinal glucose transport: Sodium-dependent glucose transporter 1

Flavonoids are a large group of natural polyphenols, which are nearly ubiquitous in plants and are, therefore, ingested by man and animals with their regular diet (Herrmann, 1988). Many flavonoids are well known for their antioxidative capabilities and are able to influence several key enzymes in vitro (Middleton et al. 2000). On the basis of epidemiological studies, a protective effect of plant flavonoids against CHD has been claimed (Hertog et al. 1993a; Rimm et al. 1996). Among the many different flavonoids, the flavonol quercetin is relatively more abundant, high concentrations of which can be found, for instance, in tea, apples and onions (Hertog et al. 1993b; Price \& Rhodes, 1997). Estimated human daily intake ranges between 10 and $20 \mathrm{mg}$ in Western populations (Hertog et al. 1993a,b; de Vries et al. 1997). In plants and plantderived foods, flavonoids are largely present as conjugates with the flavonoid aglycone linked to variable sugar moieties by a $\beta$-glycosidic bond (Kühnau, 1976).

Recent investigations have demonstrated an influence of quercetin-3-O-glucoside (Q3G) or quercetin-4'-O-glucoside (Q4G) on intestinal glucose and galactose absorption in vitro (Gee et al. 1998, 2000; Ader et al. 2001). Further studies have presented data supporting the hypothesis that Q3G and Q4G can be transported by Na-dependent glucose transporter 1 (SGLT1) present in the brush-border membrane (BBM) of the small intestine (Walgren et al. 2000b; Wolffram et al. 2002; Walle \& Walle, 2003). It is still a matter of debate as to whether mucosal uptake of these flavonol glucosides, i.e. the first step in absorption, can occur via SGLT1. The results of recent in vivo studies with rats tend to favour a different mechanism of absorption; hydrolysis of the glucosides by lactase phloridzin hydrolase (LPH; EC 3.2.1.108) followed by diffusive uptake of the aglycone quercetin into the enterocytes (Crespy et al. 2001; Sesink et al. 2003). Irrespective of these considerations, it is obvious that certain flavonol glycosides are able to interact with intestinal nutrient transport systems.

The present study was performed to examine the interaction between quercetin glycosides and Na-dependent glucose absorption in more detail, using isolated jejunal $\mathrm{BBM}$ vesicles (BBMV). In particular, we wanted to investigate whether the glucose transporter SGLT1 can transport Q3G.

\footnotetext{
Abbreviations: BBM, brush-border membrane; BBMV, brush-border-membrane vesicles; LPH, lactase phloridzin hydrolase; Q3G, quercetin-3- $O$ glucoside; Q4G, quercetin-4'-O-glucoside; SGLT1, Na-dependent glucose transporter 1.

* Corresponding author: Professor Dr S. Wolffram, fax + 49431880 1528, email wolffram@aninut.uni-kiel.de
} 


\section{Materials and methods}

\section{Preparation of brush-border-membrane vesicles and uptake experiments}

Mid-jejunum was obtained from freshly killed pigs at a local slaughterhouse. The intestine was immediately opened along the mesenteric border, cleaned with chilled saline (containing the proteinase inhibitor phenylmethylsulfonyl-fluoride $(40 \mathrm{mg} / \mathrm{l})$ and dithiothreitol $(154 \mathrm{mg} / \mathrm{l})$ ) and transported in ice-cold saline to the laboratory within 15-20 min. All subsequent isolation steps were performed at $4{ }^{\circ} \mathrm{C}$. BBMV were prepared from mucosal scrapings as described previously (Wolffram et al. 1992). The final vesicle suspension was prepared in buffers as indicated in the figure legends. Samples of $400 \mu l$ were stored under liquid $\mathrm{N}_{2}$ until use.

Purification of the BBM was routinely checked by determination of the BBM marker enzyme alkaline phosphatase (EC 3.1.3.1) (test kit; Roche Diagnostics, Mannheim, Germany). The specific alkaline phosphatase activity was several-fold (13.7 (SEM 1.4)) enriched in the final BBM fraction (247.6 (SEM 44.9) and 3233.3 (SEM 443.4) mUnits/mg protein in the original mucosa homogenate and BBM suspension, respectively; means from six preparations). Cross-contamination of these BBM preparations with basolateral membranes was negligible since the specific activity of the $\mathrm{Na}^{+} / \mathrm{K}^{+}$-ATPase, a marker enzyme for basolateral membranes (EC 3.6.1.3), was hardly altered in the final BBM fraction (102.0 (SEM 27.8) $\mathrm{mUnits} / \mathrm{mg}$ protein) compared with the activity in the mucosa homogenate (113.2 (SEM 8.9) mUnits/mg protein). Protein was determined by using the Bio-Rad protein assay kit with bovine albumin as standard (Bio-Rad-Laboratories, Munich, Germany).

Uptake of ${ }^{3} \mathrm{H}$-labelled D-glucose or ${ }^{3} \mathrm{H}$-labelled L-leucine (Amersham Pharmacia Biotech, Amersham, Bucks, UK) was determined by a rapid filtration technique. Short-term incubations (incubation time $<10 \mathrm{~s}$ ) were performed using a semi-automatic incubation device (N. Bader, Kilchberg, Switzerland) first described by Kessler et al. (1978). Unless otherwise stated, uptake was started by adding $10 \mu \mathrm{l}$ BBMV suspension to $20 \mu \mathrm{l}$ incubation medium and quenched by adding $3 \mathrm{ml}$ ice-cold stop solution of the same composition as the final reaction mixture without the addition of substrate. The diluted mixture was immediately sucked through a nitro-cellulose filter $(0.45 \mu \mathrm{m}$ pore size; Schleicher and Schüll, Dassel, Germany) and rinsed twice with the stop solution. The composition of the reaction media is indicated in the figure legends. Blanks without the addition of vesicle suspension were performed for correction of unspecific binding of radioactivity to the filters. Substrate uptake was measured at room temperature. The radioactivity remaining on the filters was determined by liquid scintillation counting (Tri Carb 2100TR; Perkin-Elmer, Rodgau-Jügesheim, Germany).

The utility of each BBMV preparation for transport studies was tested by means of cumulative $\left[{ }^{3} \mathrm{H}\right] \mathrm{D}$-glucose transport. In the presence of an inwardly directed $\mathrm{Na}^{+}$gradient (initial $\mathrm{Na}^{+}$gradient $100 \mathrm{~mm}$ ), uptake of D-glucose $(0 \cdot 1 \mathrm{mM})$ was strongly $\mathrm{Na}^{+}$dependent and showed a peak overshoot (at $1 \mathrm{~min}$ incubation):equilibrium uptake (at 60 min incubation) ratio of 12.2 (SEM 1.3) (mean from six determinations). In contrast, uptake under $\mathrm{Na}^{+}$-free conditions only slowly reached equilibrium.

\section{Calculation of kinetic parameters}

The concentration dependence of D-glucose uptake into BBMV was analysed based on the subsequent Michaelis-Menten-type equation with one active (saturable) and a diffusive component:

$$
\begin{aligned}
V= & V_{\max } \times \text { substrate concentration } / \\
& \left(K_{\mathrm{M}}+\text { substrate concentration }\right) \\
& +K_{\mathrm{D}} \times \text { substrate concentration }
\end{aligned}
$$

Where $V$ is the velocity of substrate uptake, $V_{\max }$ is maximal transport velocity, $K_{\mathrm{M}}$ is an affinity constant (50\% saturation) and $K_{\mathrm{D}}$ is a permeability constant for passive diffusion. The parameters were calculated by non-linear curve fitting using GraphPad Prism version 3.00 (GraphPad Software, San Diego, CA, USA).

\section{Determination of the stability of quercetin-3-O-glucoside}

To examine the stability of Q3G during the short-term incubation experiments, $10 \mu \mathrm{l}$ BBMV suspension were added to $20 \mu$ l incubation medium containing $0.6 \mathrm{~mm}-$ Q3G. After an incubation time of $5 \mathrm{~s}$, flavonols were extracted by the addition of $5.5 \mathrm{ml}$ acetone and horizontally shaking the samples for $20 \mathrm{~min}$. Rhamnetin $(1 \mu \mathrm{g}$ in $20 \mu \mathrm{l}$ methanol) was added to the samples as an internal standard. The further sample preparation and HPLC analysis of the flavonols have been described previously (Ader et al. 2000).

\section{Chemicals}

Standard chemicals were from Merck, Darmstadt, Germany. Methyl- $\alpha$-D-glucopyranoside and phloridzin were obtained from Sigma-Aldrich, Deisenhofen, Germany. Quercetin aglycone, Q3G, Q4G, quercetin-3-O-galactoside, quercetin-3-O-glucorhamnoside, genistein-7- $O$-glucoside, naringenin-7- $O$-glucoside and cyanidin-3,5- $O$-diglucoside were purchased from Carl Roth GmbH (Karlsruhe, Germany). ${ }^{3}$ H-labelled D-glucose and L-leucine were from Amersham Pharmacia Biotech.

\section{Statistical evaluation}

Data are presented as mean values and standard errors of the mean. The differences between two means were statistically evaluated using Student's $t$ test. Statistical comparison between more than two means was performed by the Kruskal-Wallis test with pair comparison by Dunn's post hoc test due to inhomogeneous variances (tested with the method of Bartlett). A $P$ value of less than 0.05 was considered significant. All statistical analyses of data were computed using GraphPad Instat version 3.00 (GraphPad Software). 


\section{Results}

Effect of quercetin-3-O-glucoside on glucose and leucine uptake

The time course of glucose uptake $(0.1 \mathrm{mM})$ into BBMV in the presence of an inwardly directed initial $\mathrm{Na}^{+}$gradient is shown in Fig. 1 (A). Under control conditions, glucose uptake showed a typical 'overshoot' phenomenon at short incubation times, reaching its peak value at $1 \mathrm{~min}$. This demonstrated that functioning vesicles were able to concentrate intravesicular glucose several-fold above equilibrium uptake. The presence of Q3G $(0.8 \mathrm{~mm})$ in the incubation medium significantly inhibited glucose uptake under $\mathrm{Na}^{+}$gradient conditions up to an incubation period of $1 \mathrm{~min}$. The time course of glucose uptake in the presence of an inwardly directed choline ${ }^{+}$gradient is shown in Fig. 1 (B). In the absence of $\mathrm{Na}^{+}$, glucose accumulated only slowly inside the vesicles until equilibrium conditions were reached. This $\mathrm{Na}^{+}$-independent glucose uptake, however, was also significantly inhibited by Q3G.

In order to check if the inhibitory effect of Q3G was specific for glucose uptake, the influence of the flavonol glucoside on L-leucine uptake was tested. Q3G (0.8 mM) had no significant effect on the $\mathrm{Na}^{+}$-dependent and -independent uptake of L-leucine $(0 \cdot 1 \mathrm{mM})$ into BBMV (data not shown).

Effect of quercetin-3-O-glucoside on kinetic parameters of glucose uptake

Kinetic parameters of substrate transport into BBMV can only be examined at short incubation periods when substrate uptake increases linearly and is not influenced by reflux of the substrate. In the presence of an inwardly directed $\mathrm{Na}^{+}$gradient, glucose uptake was nearly linear up to an incubation period of $10 \mathrm{~s}$ (data not shown). Thus, in the subsequent experiments an incubation period of $5 \mathrm{~s}$ was chosen.

First, effects of increasing Q3G concentrations were tested on initial glucose uptake under $\mathrm{Na}^{+}$gradient conditions. As shown in Fig. 2, the effect of Q3G was significant at concentrations $\geqq 0.4 \mathrm{~mm}$. At $0.8 \mathrm{~mm}$, inhibition was around $50 \%$. Higher concentrations of Q3G could not be tested due to its limited water solubility.

Next, initial glucose uptake as a function of the extravesicular substrate concentration was determined, either under control conditions or in the presence of $0.5 \mathrm{mM}$ Q3G (Fig. 3). In the presence of an inwardly directed $\mathrm{Na}^{+}$gradient, total glucose uptake was curvilinear under both conditions. The data were fitted to a MichaelisMenten-type equation as described on p. 850 (non-linear regression coefficient for controls, $R^{2}$ 0.9949; for Q3G, $R^{2}$ 0.9978). The calculated apparent kinetic parameters are shown in Table 1. Q3G increased the value of the constant for half-maximal glucose uptake of the saturable component significantly, whereas the maximal transport velocity was not altered. In addition, Q3G also had an impact on the non-saturable diffusive component, since it decreased the value of the permeability constant for passive diffusion significantly.

\section{Determination of quercetin-3-O-glucoside stability}

It was checked whether Q3G was hydrolysed by $\beta$-glucosidases of the BBM under experimental conditions. For this experiment, incubation conditions were identical to the measurement of initial glucose uptake. Q3G was added to the transport buffer at a concentration of
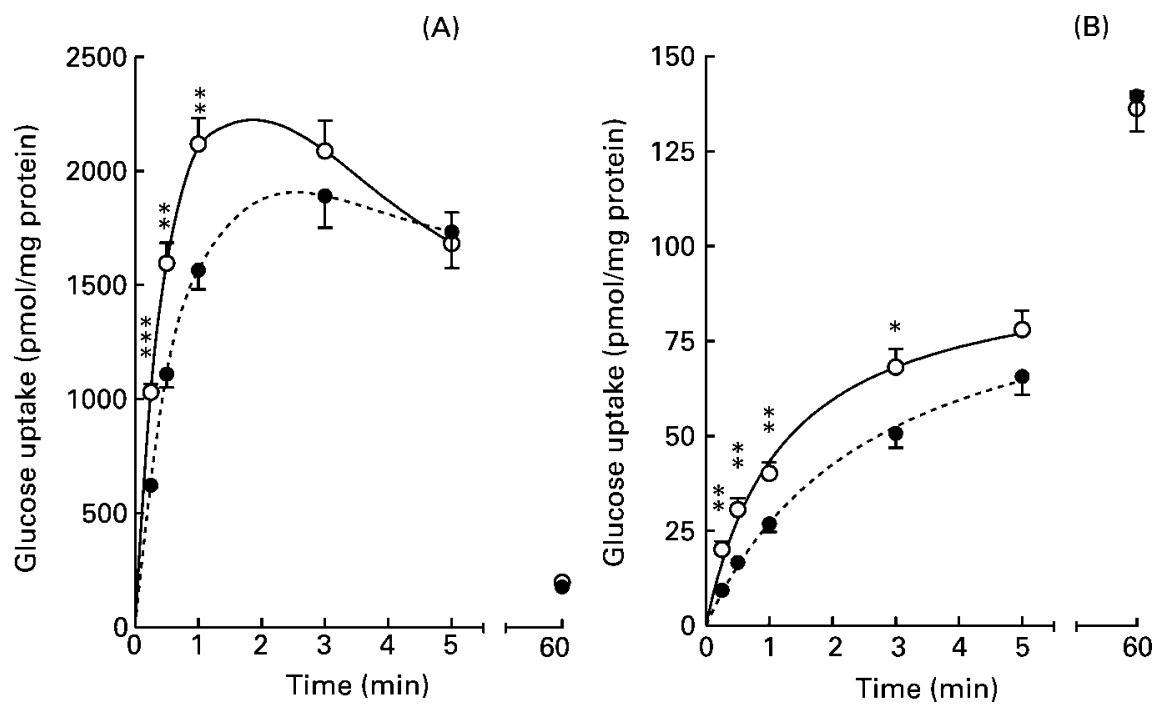

Fig. 1. Effect of quercetin-3-O-glucoside (Q3G) on $\mathrm{Na}^{+}$-dependent $(\mathrm{A})$ and -independent $(\mathrm{B})$ uptake of $\mathrm{D}$-glucose into brush-border-membrane vesicles. Values are means of six determinations from three different vesicle preparations, each determined in duplicate, with standard errors of the mean represented by vertical bars. Where absent, the standard error of the mean was smaller than the symbol. Vesicles were prepared in (mM): D-mannitol, 300; $\mathrm{MgSO}_{4} .7 \mathrm{H}_{2} \mathrm{O}, 0.1$; HEPES, 35, adjusted with Tris(hydroxymethyl)-amino methane (Tris) to $\mathrm{pH} 7.2$ and incubated in a transport buffer. Resulting final concentrations were $(\mathrm{mM}):{ }^{3} \mathrm{H}$-labelled D-glucose, 0.1 ; D-mannitol, 100; $\mathrm{NaCl}, 100(\mathrm{~A})$ or choline chloride, 100 (B), respectively; $\mathrm{MgSO}_{4} .7 \mathrm{H}_{2} \mathrm{O}, 0 \cdot 1$; HEPES-Tris ( $\mathrm{pH} 7 \cdot 2$ ), 35. Incubations were performed under control conditions (O) or in the presence of $0.8 \mathrm{~mm}-\mathrm{Q} 3 \mathrm{G}(\bullet)$. Mean values for vesicles incubated with Q3G were significantly different from those for controls: ${ }^{*} P<0.05,{ }^{* \star} P<0.01$, ${ }^{* * \star} P<0.001$. 


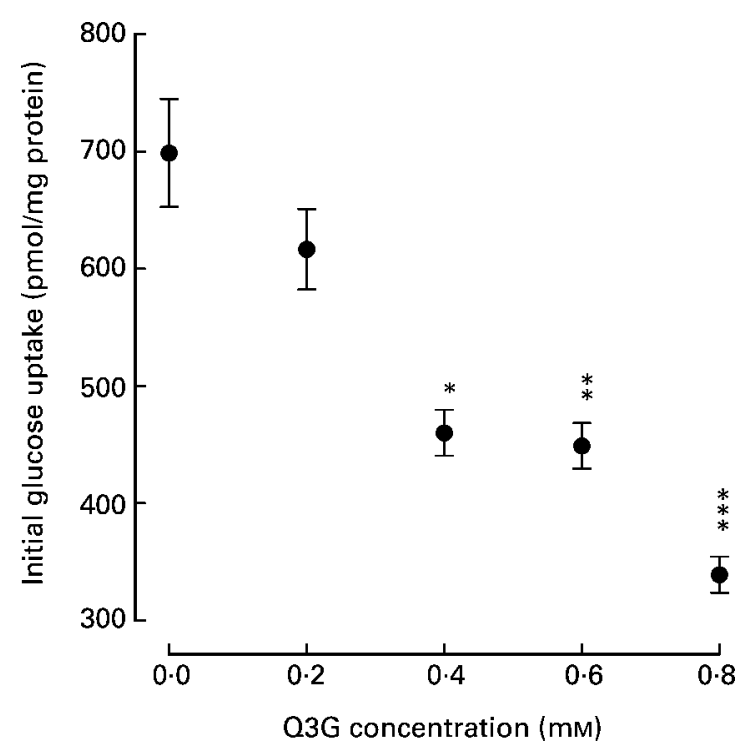

Fig. 2. Effect of increasing quercetin-3-O-glucoside (Q3G) concentrations on initial glucose uptake in the presence of an inwardly directed $\mathrm{Na}^{+}$gradient. Values are means of nine determinations from three different vesicle preparations, each determined in triplicate, with standard errors of the mean represented by vertical bars. Vesicles were prepared in (mM): D-mannitol, 300; $\mathrm{MgSO}_{4} .7 \mathrm{H}_{2} \mathrm{O}$, 0.1 ; HEPES, 35, adjusted with Tris(hydroxymethyl)-amino methane (Tris) to $\mathrm{pH} 7.2$ and incubated in a transport buffer. Resulting final concentrations were (mM): ${ }^{3} \mathrm{H}$-labelled D-glucose, 0.1; D-mannitol, 100; $\mathrm{NaCl}, 100 ; \mathrm{MgSO}_{4} .7 \mathrm{H}_{2} \mathrm{O}, 0.1$; HEPES-Tris (pH 7.2), 35; Q3G, indicated concentrations. The incubation period was $5 \mathrm{~s}$. D-Glucose uptake was significantly inhibited by Q3G: ${ }^{*} P<0.05$, ${ }^{\star *} P<0.01,{ }^{* \star *} P<0.001$

$0.6 \mathrm{~mm}$. The appearance of the aglycone quercetin was determined after an incubation time of $5 \mathrm{~s}$. After this incubation period, quercetin concentration was $1 \cdot 15$ (SEM $0 \cdot 16$ ) $\mu \mathrm{M}(n 3)$, indicating that only $0.2 \%$ of the initially present Q3G was deglucosylated.

\section{Effects of other flavonoid glycosides on glucose uptake}

Besides Q3G, the effect of other flavonoids or glucose analogues on initial glucose uptake was investigated. The results are shown in Fig. 4. The non-metabolisable

Table 1. Apparent kinetic parameters for glucose uptake into brush-border-membrane vesicles in the presence or absence of quercetin-3-O-glucoside (Q3G) $(0.5 \mathrm{~mm}) \dagger$

(Mean values and standard errors of the mean)

\begin{tabular}{|c|c|c|c|c|c|c|}
\hline & \multicolumn{2}{|c|}{$\begin{array}{r}V_{\max }(\mathrm{nmol} / \mathrm{mg} \\
\text { protein per } 5 \mathrm{~s})\end{array}$} & \multicolumn{2}{|c|}{$K_{\mathrm{M}}(\mathrm{mmol} / \mathrm{l})$} & \multicolumn{2}{|c|}{$\begin{array}{c}K_{\mathrm{D}}(\mu \mathrm{l} / \mathrm{mg} \\
\text { protein per } 5 \mathrm{~s})\end{array}$} \\
\hline & Mean & SEM & Mean & SEM & Mean & SEM \\
\hline Control & $2 \cdot 1$ & 0.4 & $0 \cdot 17$ & 0.01 & 0.25 & 0.04 \\
\hline Q3G & $2 \cdot 0$ & 0.4 & $0.32^{\star}$ & 0.03 & $0 \cdot 12^{*}$ & 0.01 \\
\hline
\end{tabular}

$V_{\max }$, maximal transport velocity of saturable component; $K_{\mathrm{M}}$, affinity constant of saturable component (50\% saturation); $K_{\mathrm{D}}$, permeability constant of non-saturable component.

${ }^{*}$ Mean values were significantly different from the control values $(P<0.05)$.

† Values were calculated from the data shown in Fig. 3 (for details, see legend of Fig. 3)

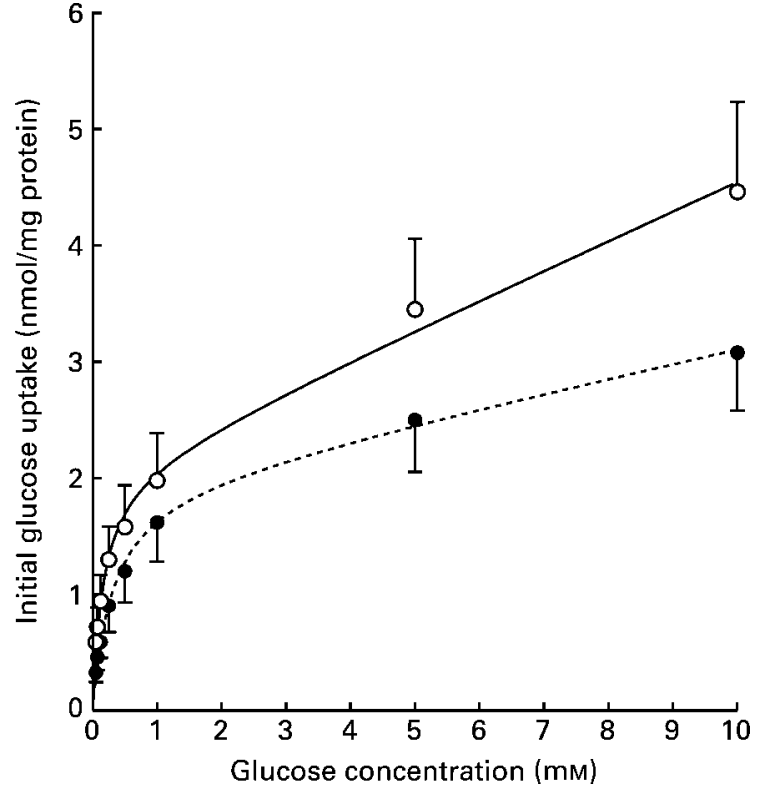

Fig. 3. Effect of quercetin-3-O-glucoside (Q3G) on transport kinetics of glucose uptake in the presence of an inwardly directed $\mathrm{Na}^{+}$ gradient. Values are means of nine determinations from three different vesicle preparations, each determined in triplicate, with standard errors of the mean represented by vertical bars. Vesicles were prepared in (mM): D-mannitol, 300; $\mathrm{MgSO}_{4} 7 \mathrm{H}_{2} \mathrm{O}, 0.1$; HEPES, 35, adjusted with Tris(hydroxymethyl)-amino methane (Tris) to $\mathrm{pH} 7.2$ and incubated in a transport buffer. Resulting final concentrations were (mM): D-mannitol, $100 ; \mathrm{NaCl}, 100 ; \mathrm{MgSO}_{4} .7 \mathrm{H}_{2} \mathrm{O}, 0.1$; HEPES-Tris (pH 7.2), 35; ${ }^{3} \mathrm{H}$-labelled D-glucose, indicated concentrations. Incubations were performed under control conditions $(O)$ or in the presence of $0.5 \mathrm{~mm}-\mathrm{Q} 3 \mathrm{G}(\bullet)$; the incubation period was $5 \mathrm{~s}$.

glucose analogue methyl- $\alpha$-D-glucopyranoside significantly inhibited glucose uptake, whereas the SGLT1 inhibitor phloridzin nearly abolished it. Among the tested flavonoids, only the quercetin glucosides Q3G and Q4G inhibited glucose uptake. The galactoside as well as the glucorhamnoside and the aglycone form of quercetin were ineffective. The investigated glucosides of the isoflavone genistein, the flavanone naringenin and of the anthocyanidine cyanidin were also not effective.

Trans-stimulation of glucose uptake by quercetin-3-Oglucoside

To investigate if $\mathrm{Q} 3 \mathrm{G}$ itself was transported into the vesicles via SGLT1, trans-stimulation experiments were performed. These experiments were executed under $\mathrm{Na}^{+}$ equilibrium conditions to eliminate any stimulation of glucose uptake by an $\mathrm{Na}^{+}$gradient. Vesicles were prepared in a solution containing $0.5 \mathrm{mM}-\mathrm{Q} 3 \mathrm{G}$ and the volume of the incubation buffer was increased to $190 \mu \mathrm{l}$ in order to achieve an outwardly directed Q3G gradient of 20:1. As shown in Fig. 5, this outwardly directed Q3G gradient significantly increased initial glucose uptake after an incubation period of $5 \mathrm{~s}$.

\section{Discussion}

The present study showed that the quercetin monoglucosides Q3G and Q4G inhibited $\mathrm{Na}^{+}$-dependent D-glucose 


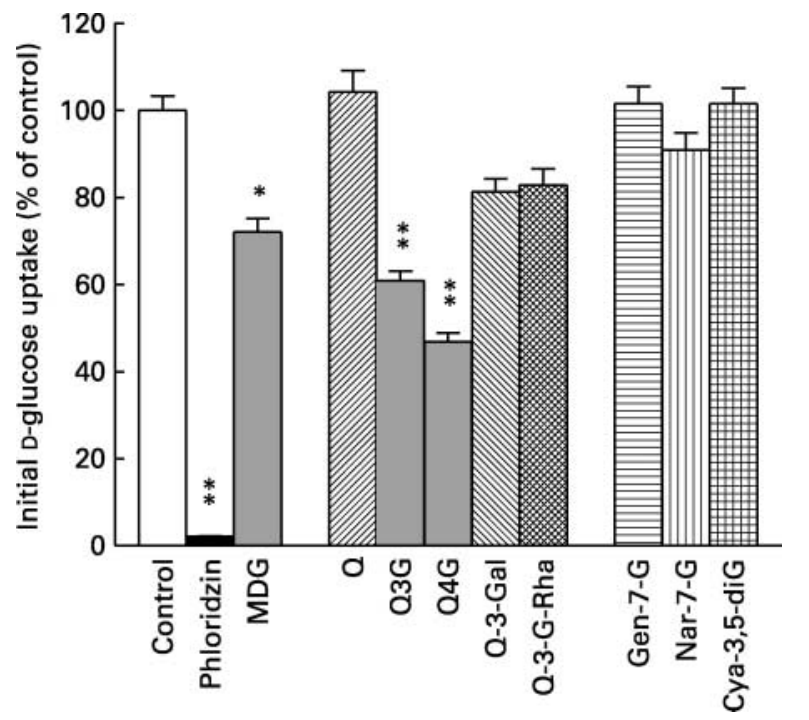

Fig. 4. Effect of different flavonoid glycosides on glucose uptake in the presence of an inwardly directed $\mathrm{Na}^{+}$gradient. Values are means of nine determinations from three different vesicle preparations, each determined in triplicate, with standard errors of the mean represented by vertical bars. Vesicles were prepared in (mм): D-mannitol, 300; $\mathrm{MgSO}_{4} .7 \mathrm{H}_{2} \mathrm{O}, 0.1$; HEPES, 35, adjusted with Tris (hydroxymethyl)-amino methane (Tris) to $\mathrm{pH} 7.2$ and incubated in a transport buffer. Resulting final concentrations were (mM): D-mannitol, $100 ; \mathrm{NaCl}, 100 ; \mathrm{MgSO}_{4} .7 \mathrm{H}_{2} \mathrm{O}, 0.1$; HEPES-Tris (pH 7.2), 35 (controls). Experimental vesicles were also incubated with one of the following compounds (each at a concentration of $0.6 \mathrm{mM}$ ): phloridzin; methyl- $\alpha$-D-glucopyranoside (MDG); quercetin aglycone $(Q)$; quercetin-3-O-glucoside (Q3G); quercetin-4'-O-glucoside (Q4G); quercetin-3-O-galactoside (Q-3-Gal); quercetin-3-O-glucorhamnoside (Q-3-G-Rha); genistein-7-O-glucoside (Gen-7-G); naringenin7-O-glucoside (Nar-7-G); cyanidin-3,5-O-diglucoside (Cya-3,5-diG). The incubation period was $5 \mathrm{~s}$. Mean values were significantly different from those for controls: ${ }^{\star} P<0.01,{ }^{\star}{ }^{\star} P<0.001$.

uptake into BBMV of pig jejunum, which is mediated via SGLT1. The inhibition was specific for SGLT1, since Q3G did not affect $\mathrm{Na}^{+}$-dependent uptake of L-leucine. This observation confirms earlier studies, which suggested an interaction of Q3G and Q4G with $\mathrm{Na}^{+}$-dependent glucose or galactose absorption in the small intestine (Gee et al. 1998, 2000; Ader et al. 2001; Wolffram et al. 2002). While Q3G did not alter the maximal transport capacity of $\mathrm{Na}^{+}$-dependent glucose uptake, it increased the affinity constant of this saturable transport mechanism. In agreement with a previous study performed with intact mucosal jejunal pieces (Ader et al. 2001), this clearly indicates a competitive type of inhibition.

Quercetin glucosides can be hydrolysed by the glycosidase LPH located in the BBM (Ioku et al. 1998; Day et al. 2000). Glucose, potentially liberated from Q3G due to enzymic or spontaneous hydrolysis, could have been responsible for the observed inhibitory effect on SGLT1. Thus, we checked the stability of Q3G under our experimental conditions. However, only $0.2 \%$ of the original amount of Q3G was recovered as quercetin aglycone, i.e. hardly any hydrolysis had occurred. We, thus, conclude that intact Q3G was the molecule responsible for the competitive inhibition of SGLT1.

In the trans-stimulation experiments, an outwardly directed Q3G gradient stimulated initial glucose uptake

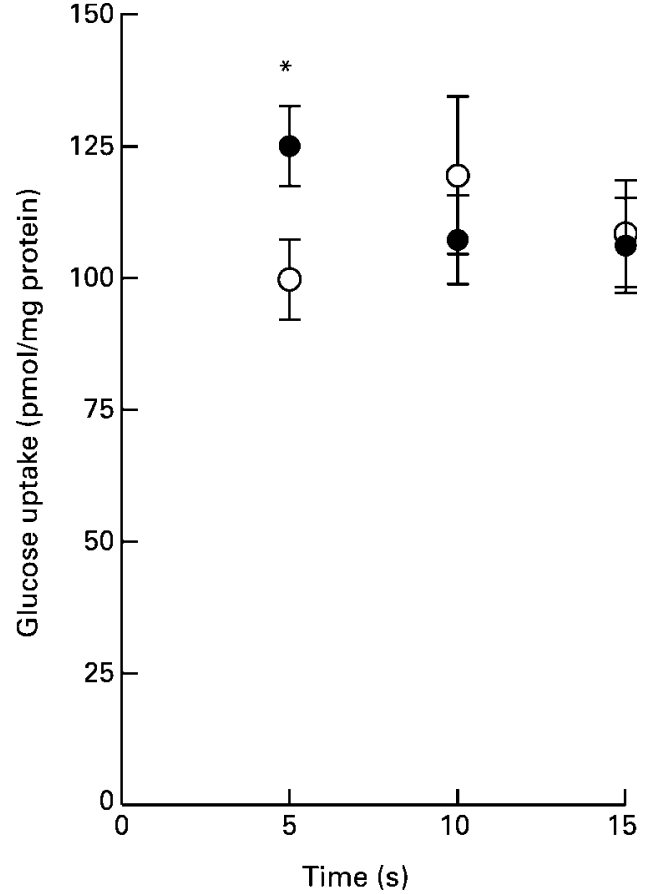

Fig. 5. Effect of an outwardly directed quercetin-3-O-glucoside (Q3G) gradient on glucose uptake. Values are means from three different vesicle preparations, each determined in triplicate, with standard errors of the mean represented by vertical bars. Vesicles were prepared in (mM): D-mannitol, $100 ; \mathrm{NaCl}, 100 ; \mathrm{MgSO}_{4} .7 \mathrm{H}_{2} \mathrm{O}$, 0.1 ; HEPES, 35, without $(O)$ or with $0.5 \mathrm{~mm}-\mathrm{Q} 3 \mathrm{G}(\bullet)$ adjusted with Tris(hydroxymethyl)-amino methane (Tris) to $\mathrm{pH} 7.2$ and incubated in a transport buffer. Resulting final concentrations were (mM): ${ }^{3} \mathrm{H}$-labelled D-glucose, 0.1; D-mannitol, 100; $\mathrm{NaCl}, 100$; $\mathrm{MgSO}_{4} .7 \mathrm{H}_{2} \mathrm{O}, 0.1$; HEPES-Tris $(\mathrm{pH} 7.2), 35$, without $(\mathrm{O})$ or with $0.025 \mathrm{~mm}-\mathrm{Q} 3 \mathrm{G}(\bullet)$. ${ }^{*}$ Mean value of vesicles incubated with Q3G was significantly different from that for the control $(P<0.05)$.

into BBMV. The observation that after longer incubation periods this stimulatory effect faded can be explained by a fast dissipation of the Q3G gradient due to the efflux of the glucoside. Efflux of the monoglucoside could have been mediated by multidrug resistance-associated proteins as demonstrated for Q4G (Walgren et al. 2000a; Walle $\&$ Walle, 2003). Together with the competitive cis inhibition of glucose uptake by Q3G, these trans-stimulation experiments suggest that $\mathrm{Q} 3 \mathrm{G}$ itself is transported by SGLT1 (Wilbrandt \& Rosenberg, 1961). The present findings are further supported by a recent study demonstrating the transport of Q4G via SGLT1 in Chinese hamster ovary cells (Walle \& Walle, 2003). However, the present experiments do not allow an estimate for the proportion of SGLT1-mediated uptake of overall Q3G absorption.

In contrast to the present findings at the level of SGLT1, another group demonstrated that $\mathrm{Q} 3 \mathrm{G}$ did not inhibit overall glucose absorption in rat small intestine perfused in situ (Crespy et al. 2001). Phloridzin, which inhibits SGLT1 as well as LPH, failed to influence Q3G absorption in that study. However, the Q3G dosage used in those experiments was much lower compared with that for phloridzin (Crespy et al. 2001). In a similar intestinal perfusion model, Sesink et al. (2003) used a more selective inhibitor of LPH, which does not affect SGLT1. Since this inhibitor hampered about $75 \%$ of the intestinal Q3G absorption, the authors 
came to the conclusion that hydrolysis of Q3G by LPH was a prerequisite for the major part of the Q3G absorption in the small intestine. However, they did not rule out that the remaining part could be due to the uptake of intact Q3G by SGLT1 (Sesink et al. 2003). With regard to the results of the present study, it seems probable that at least a certain part of Q3G is transported by SGLT1. It should be mentioned, however, that another group presented results that argue against the involvement of SGLT1 in the intestinal absorption of Q3G (Day et al. 2003).

In the present study, $\mathrm{Na}^{+}$-dependent glucose uptake was inhibited only by the quercetin monoglucosides Q3G and $\mathrm{Q} 4 \mathrm{G}$, whereas the aglycone and the glucorhamnoside were ineffective. Even the galactoside failed to influence $\mathrm{Na}^{+}$-dependent glucose absorption, although galactose itself is a good substrate for SGLT1. Glucosides from several other flavonoid classes (naringenin, genistein, cyanidin) also failed to affect this glucose transporter. Thus, inhibition of $\mathrm{Na}^{+}$-dependent glucose absorption seems to be rather specific to quercetin monoglucosides. A previous study described inhibition of SGLT1 in chicken enterocytes by another glucoside of naringenin, namely 7-methoxy-naringenin-5-glucoside (Kimmich \& Randles, 1978). However, this inhibition was only very weak and probably not significant. In contrast, aglycones from several flavonoid subclasses inhibited effectively $\mathrm{Na}^{+}$-independent uptake of a glucose analogue in the same study, probably acting on the glucose transporter GLUT2 (Kimmich \& Randles, 1978).

Interestingly, we found evidence that Q3G also inhibited SGLT1-independent, non-saturable uptake of glucose. This uptake component could be due to passive glucose diffusion or to glucose transport via a $\mathrm{Na}^{+}$-independent glucose carrier, which does not saturate at the glucose concentrations used in the present study. It is rather improbable that Q3G altered the permeability of the BBM, thus inhibiting unspecific diffusion of glucose. In contrast to the lipophilic aglycone quercetin, hydrophilic quercetin glycosides appear to be unable to interact with lipid membranes (Saija et al. 1995). In addition, leucine transport was not affected by $\mathrm{Q} 3 \mathrm{G}$. On the other hand, it is reasonable to speculate that Q3G affected another glucose transporter. A recent study described a low-affinity and high-capacity transport system for glucose in pig jejunum (Halaihel et al. 1999). This novel $\mathrm{Na}^{+}$-independent saturable transport system was distinct from SGLT1 and the known intestinal hexose transporters GLUT2 and GLUT5. It could only be differentiated from passive diffusion at very high glucose concentrations greater than $50 \mathrm{~mm}$, because at lower substrate concentrations glucose uptake still increased linearly (Halaihel et al. 1999). Thus, the observed inhibition of the 'non-saturable' component of glucose uptake in the present study could refer to such a $\mathrm{Na}^{+}$-independent, low-affinity saturable transport system.

Quercetin aglycone and other flavonoid aglycones were shown to inhibit 2-deoxyglucose transport via GLUT2 as well as ascorbic acid transport via the intestinal vitamin $\mathrm{C}$ transporter in Xenopus oocytes (Song et al. 2002). In rats, orally applied quercetin was able to inhibit glucose and ascorbic acid absorption in vivo (Song et al. 2002). In this context, one has to keep in mind that quercetin aglycone might be released from its glucosides by LPH on the luminal side of the BBM (Day et al. 1998, 2000; Ioku et al. 1998).

It appears improbable that the small amounts of quercetin monoglucosides normally ingested with food inhibit SGLT1-mediated glucose absorption in vivo. However, uptake via SGLT1 could explain the higher bioavailability of these monoglucosides compared with the aglycone or to more complex quercetin glycosides in various species (Hollman et al. 1999; Crespy et al. 2001; Cermak et al. 2003). The reasons for this higher bioavailability are still a matter of debate. Some authors ascribe it to the activity of LPH, but the question of varying activities of this enzyme, which is physiologically down regulated in many adult individuals, has not been adequately addressed until now.

Taken together, we have shown that Q3G inhibits glucose uptake by SGLT1 in a specific and competitive manner. Q3G also inhibited $\mathrm{Na}^{+}$-independent glucose uptake. Furthermore, Q3G seems to be transported by SGLT1. Thus, at least part of the intestinal Q3G absorption could occur via this route.

\section{Acknowledgements}

We would like to thank Beat Grenacher for his help with the isolation of the vesicles. The present study was supported by a grant of the Deutsche Forschungsgemeinschaft (WO 763/2-1).

\section{References}

Ader P, Blöck M, Pietzsch S \& Wolffram S (2001) Interaction of quercetin glucosides with the intestinal sodium/glucose cotransporter (SGLT-1). Cancer Lett 162, 175-180.

Ader P, Wessmann S \& Wolffram S (2000) Bioavailability and metabolism of the flavonol quercetin in the pig. Free Radic Biol Med 28, 1056-1067.

Cermak R, Landgraf S \& Wolffram S (2003) The bioavailability of quercetin in pigs depends on the glycoside moiety and on dietary factors. J Nutr 133, 2802-2807.

Crespy V, Morand C, Besson C, Manach C, Demigné C \& Rémésy C (2001) Comparison of the intestinal absorption of quercetin, phloretin and their glucosides in rats. $J$ Nutr 131, 2109-2114.

Day AJ, Cañada FJ, Díaz JC, Kroon PA, Mclauchlan R, Faulds CB, Plumb GW, Morgan MR \& Williamson G (2000) Dietary flavonoid and isoflavone glycosides are hydrolysed by the lactase site of lactase phlorizin hydrolase. FEBS Lett $\mathbf{4 6 8}$, $166-170$.

Day AJ, Dupont MS, Ridley S, Rhodes M, Rhodes MJ, Morgan MR \& Williamson G (1998) Deglycosylation of flavonoid and isoflavonoid glycosides by human small intestine and liver beta-glucosidase activity. FEBS Lett 436, 71-75.

Day AJ, Gee JM, Dupont MS, Johnson IT \& Williamson G (2003) Absorption of quercetin-3-glucoside and quercetin-4'-glucoside in the rat small intestine: the role of lactase phlorizin hydrolase and the sodium-dependent glucose transporter. Biochem Pharmacol 65, 1199-1206.

de Vries JHM, Janssen PL, Hollman PCH, van Staveren WA \& Katan MB (1997) Consumption of quercetin and kaempferol in free-living subjects eating a variety of diets. Cancer Lett 114, $141-144$. 
Gee JM, Dupont MS, Day AJ, Plumb GW, Williamson G \& Johnson IT (2000) Intestinal transport of quercetin glycosides in rats involves both deglycosylation and interaction with the hexose transport pathway. J Nutr 130, 2765-2771.

Gee JM, Dupont MS, Rhodes MJC \& Johnson IT (1998) Quercetin glucosides interact with the intestinal glucose transport pathway. Free Radic Biol Med 25, 19-25.

Halaihel N, Gerbaud D, Vasseur M \& Alvarado F (1999) Heterogeneity of pig intestinal D-glucose transport systems. Am J Physiol 277, C1130-C1141.

Herrmann K (1988) On the occurence of flavonol and flavone glycosides in vegetables. $Z$ Lebensm Unters Forsch 186, 1-5.

Hertog MGL, Feskens EJ, Hollman PCH, Katan MB \& Kromhout D (1993a) Dietary antioxidant flavonoids and risk of coronary heart disease: the Zutphen Elderly Study. Lancet 342, 1007-1011.

Hertog MGL, Hollman PCH, Katan MB \& Kromhout D (1993b) Intake of potentially anticarcinogenic flavonoids and their determinants in adults in The Netherlands. Nutr Cancer 20 , $21-29$.

Hollman PCH, Bijsman MNCP, van Gameren Y, Cnossen EPJ, de Vries JHM \& Katan MB (1999) The sugar moiety is a major determinant of the absorption of dietary flavonoid glycosides in man. Free Radic Res 31, 569-573.

Ioku K, Pongpiriyadacha Y, Konishi Y, Takei Y, Nakatani N \& Terao J (1998) $\beta$-Glucosidase activity in the rat small intestine toward quercetin monoglucosides. Biosci Biotechnol Biochem 62, 1428-1431.

Kessler M, Tannenbaum V \& Tannenbaum C (1978) A simple apparatus for performing short-time (1-2 seconds) uptake measurements in small volumes; its application to D-glucose transport studies in brush border vesicles from rabbit jejunum and ileum. Biochim Biophys Acta 509, 348-359.

Kimmich GA \& Randles J (1978) Phloretin-like action of bioflavonoids on sugar accumulation capability of isolated intestinal cells. Membr Biochem 1, 221-237.

Kühnau J (1976) The flavonoids. A class of semi-essential food components: their role in human nutrition. World Rev Nutr Diet 24, 117-191.

Middleton E, Kandaswami C \& Theoharides TC (2000) The effects of plant flavonoids on mammalian cells: implications for inflammation, heart disease, and cancer. Pharmacol Rev 52, 673-751.

Price KR \& Rhodes MJC (1997) Analysis of the major flavonol glycosides present in four varieties of onion (Allium cepa) and changes in composition resulting from autolysis. $J$ Sci Food Agric 74, 331-339.

Rimm EB, Katan MB, Ascherio A, Stampfer MJ \& Willett WC (1996) Relation between intake of flavonoids and risk for coronary heart disease in male health professionals. Ann Intern Med 125, 384-389.

Saija A, Scalese M, Lanza M, Marzullo D, Bonina F \& Castelli F (1995) Flavonoids as antioxidant agents: importance of their interaction with biomembranes. Free Radic Biol Med 19, 481-486.

Sesink ALA, Arts ICW, Faassen-Peters M \& Hollman PCH (2003) Intestinal uptake of quercetin-3-glucoside in rats involves hydrolysis by lactase phlorizin hydrolase. $J$ Nutr 133, 773-776.

Song J, Kwon O, Chen S, Daruwala R, Eck P, Park JB \& Levine M (2002) Flavonoid inhibition of sodium-dependent vitamin C transporter 1 (SVCT1) and glucose transporter isoform 2 (GLUT2), intestinal transporters for vitamin $\mathrm{C}$ and glucose. J Biol Chem 277, 15252-15260.

Walgren RA, Karnaky KJ, Lindenmayer GE \& Walle T (2000a) Efflux of dietary flavonoid quercetin $4^{\prime}-\beta$-glucoside across human intestinal Caco-2 cell monolayers by apical multidrug resistance-associated protein-2. J Pharmacol Exp Ther 294, 830-836.

Walgren RA, Lin JT, Kinne RKH \& Walle T (2000b) Cellular uptake of dietary flavonoid quercetin 4 '- $\beta$-glucoside by sodium-dependent glucose transporter SGLT1. J Pharmacol Exp Ther 294, 837-843.

Walle T \& Walle UK (2003) The $\beta$-D-glucoside and sodiumdependent glucose transporter 1 (SGLT1)-inhibitor phloridzin is transported by both SGLT1 and multidrug resistance-associated proteins 1/2. Drug Metab Dispos 31, 1288-1291.

Wilbrandt W \& Rosenberg T (1961) The concept of carrier transport and its corollaries in pharmacology. Pharmacol Rev 13, 109-183.

Wolffram S, Blöck M \& Ader P (2002) Quercetin-3-glucoside is transported by the glucose carrier SGLT1 across the brush border membrane of rat small intestine. J Nutr 132, 630-635.

Wolffram S, Hagemann C, Grenacher B \& Scharrer E (1992) Characterization of the transport of tri- and dicarboxylates by pig intestinal brush-border membrane vesicles. Comp Biochem Physiol 101A 759-767. 